\title{
A new Li-ion battery charger with charge mode selection based on 0.18 um CMOS for phone applications
}

\author{
Fouad Farah ${ }^{1}$, Mustapha El Alaoui ${ }^{1}$ Abdellali Elboutahiri ${ }^{1}$, Mounir Ouremchi ${ }^{1}$, Karim El Khadiri ${ }^{2}$, \\ Ahmed Tahiri², Hassan Qjidaa ${ }^{1}$ \\ 1,2,3,4,7 Department of Physics FSDM, Sidi Mohamed Ben Abbellah University, Fez, Morocco \\ ${ }^{5,6}$ Laboratory of Computer Science and Interdisciplinary Physics, ENS, Sidi Mohamed Ben Abdellah University, \\ Fez, Morocco
}

\section{Article Info \\ Article history: \\ Received Sep 11, 2020 \\ Revised Dec 10, 2020 \\ Accepted Jan 8, 2021 \\ Keywords: \\ Charge mode-selection \\ High efficiency \\ Li-ion battery \\ Temperature detector}

\begin{abstract}
A new architecture of Li-Ion battery charger with charge mode selection is presented in this work. To ensure high efficiency, good accuracy and complete protection mode, we propose an architecture based on variable current source, temperature detector and power control. To avoid the risk of damage, the Li- Ion batteries charging process must change between three modes of current (trickle current (TC), constant current (CC), and constant voltage $(\mathrm{CV}))$ in order to charge the battery with degrading current. However, the interest of this study is to develop a fast battery charger with high accuracy that is able to switch between charging modes without reducing its power efficiency, and to guarantee a complete protection mode. The proposed charger circuit is designed to control the charging process in three modes using the charging mode selection. The obtained results show that the Li-ion batteries can be successfully charged in a short time without reducing their efficiency. The proposed charger is implemented in $180 \mathrm{~nm}$ CMOS technology with a maximum charging current equal to $1 \mathrm{~A}$ and a maximum battery voltage equal to $4.22 \mathrm{~V}$, (with input range $2.7-4.5 \mathrm{~V}$ ). The chip area is $1.5 \mathrm{~mm}^{2}$ and the power efficiency is $90.09 \%$.
\end{abstract}

This is an open access article under the CC BY-SA license.

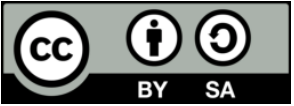

\section{Corresponding Author:}

Fouad Farah

Department of Physics FSDM

Sidi Mohamed Ben Abbellah University

Fez, 30050, Morocco

Email: fouad.farah@usmba.ac.ma

\section{INTRODUCTION}

Currently, the batteries production has encountered a revolution regarding its design, especially after the appearance of lithium-ion batteries. These batteries present a favourable choice for several devices such as phones, EDAS and electric vehicles. This is due to many reasons: weight ( Lithium-ion batteries are three times lighter than lead batteries for the same stored energy), high efficiency, the lithium batteries have a performance close to $100 \%$, Life cycle over 1000 cycles, output voltage (ranges 2.5-4.2 V), environmental impact and cost of stored energy [1].

To this day, many Li-Ion battery charger architectures have been published in the literature using CMOS technology [2-14]. As already stated, with these high performances, li-ion battery chargers became the most used for mobile applications. Thereafter, there are two architectures of battery chargers, one based on the linear regulator and the other on the switching regulator. The battery charger is implemented with CMOS technology and is integrated in a System on Chip to reduce the effect of noise and ripple. The linear 
regulator architecture represents a good choice for mobile applications compared to the switching regulator and that is due to several factors: Low cost, small size, high integration, accuracy and high power efficiency.

To improve the behaviour of linear regulator battery chargers, many studies have been proposed. The approach proposed by Ziadi and Qjidaa in [2], describes how the mode charging is accomplished using a power transistor control made by two current sources Iref1 and Iref 2 passed through a level shifter. The transistor provides a constant current to order the trickle constant current and the fast-constant current modes. However, this architecture suffers from low power efficiency due to the time lag between the power supply and the battery voltage $\mathrm{V}_{\text {BAT. }}$. Another architecture is proposed in [7, 11], it is based on the DC/DC converter. This kind of architecture is characterized by high power efficiency; however, it is not suitable for single chip integration because of its low accuracy and big area. A battery charger based on charge pump is introduced in [12-14], in spite of its low power consumption, the efficiency and accuracy of this architecture are not high enough for battery charging. If we look at the design of all of these architectures, we notice that a very important safety factor is missing which is temperature monitoring. During the charging process, the circuit temperature varies according to the state of charge or to the variation of the power supply. Thus, it is necessary to control the circuit temperature. According to the analysis of the different architectures cited in the literature regarding Li-ion battery charger, the major problems occur are low efficiency and accuracy, large surface area and temperature control.

In this study, we propose high efficiency, low power consumption and high maximum current linear for the battery charger architecture. Therefore, to extend the run time and reduce the risk of battery damage, it is necessary to control the operation of charging in the three modes of charging with degrading current [15-17]. The proposed architecture is based on variable current source and power control to keep the difference between the power and the battery voltage small and constant. In addition, a charging mode selection is proposed using a logic circuit to control each mode of charging in order to reduce power dissipation and achieve high power efficiency. A temperature detector is proposed to guarantee the safety of all circuits from temperature damage during the charging process.

\section{DESCRIPTION OF CHARGING CIRCUIT}

The proposed charging mode of a Li-ion battery charger is shown in Figure 1, the charging process of a Li-ion battery is processed into three charging states, TC, LC and $\mathrm{CV}$, with temperature detector in each state. To avoid risks due to high charging current on the battery, the charging process is treated as follows:

- If the voltage $\mathrm{V}_{\mathrm{BAT}}$ is lower than the defined voltage $\mathrm{V}_{\mathrm{L}}$ (normally $2.9 \mathrm{~V}$ ), the charger starts from the TC state with a low charging current $(200 \mathrm{~mA})$.

- If the voltage value of VBAT is between the higher voltage VH and the lower voltage VL, the charger operates at the LC state with a constant and large driving current until the value of VBAT becomes higher than $\mathrm{VH}$ (normally $4.2 \mathrm{~V}$ ), which is a predefined transition voltage.

- If the voltage value VBAT is higher than the voltage $\mathrm{VH}$, the charging process switches from the LC phase to the $\mathrm{CV}$ state. The charger then charges the battery by degrading the current to full capacity until the process stops.

Finally, to stop the charging, there are many ways: The charger stops the charging process once it detects the minimum charging current at the CV phase (charging current is reduced to the specified range). Or by the definition of the maximum charging time $[18,19]$. In our work, we used two methods at the same time to respect all charge procedures and guarantee battery protection. The charge stops when the charging current is reduced to $0.05 \mathrm{C}$, or if the temperature detected by the PTAT circuit detects 115 degrees.

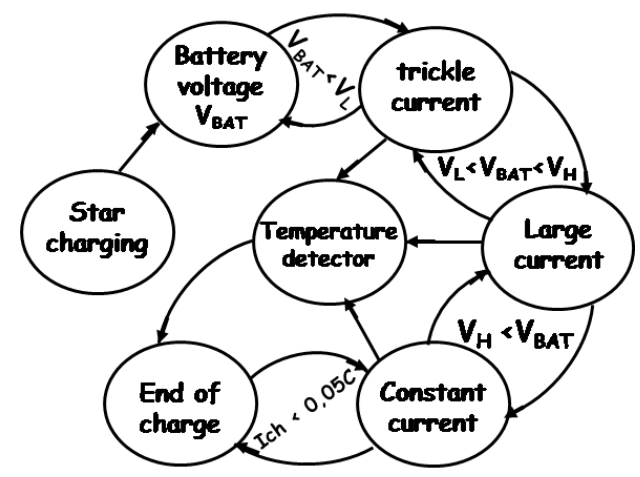

Figure 1. The proposed charging mode of a Li-ion battery charger 
The architecture of the Li-Ion battery charger system consists mainly of two blocks: The charging block and the Power supply block. For the conventional Li-Ion battery chargers, the power supply block is an AC-DC converter, which is independent of load circuitry, usually the SC or LDO. The efficiency of the conventional Li-Ion battery charger is presented in (1) [20]:

$$
\eta_{1} \cong \frac{V_{B A T} I_{C H}}{V_{D D}\left(I_{q}+I_{C H}\right)} \cong \frac{V_{B A T}}{V_{D D}}
$$

However, if we compare the quiescent current (Iq) and the charging current (Ich), we can distinguish that Iq is much smaller than Ich. Consequently, the efficiency is equal to $\mathrm{V}_{\mathrm{BAT}}, \mathrm{V}_{\mathrm{DD}}$ ratio. The $\eta 1$ is quite weak in the initial process $\left(\mathrm{V}_{\mathrm{DD}}\right.$ constant), which represents a major disadvantage. However, the variable current source circuit shown in Figure 2 can produce an adaptive reference voltage in order to retain the charger at high power throughout the charging process. The energy efficiency of the variable current source battery charger is shown in (2), it is optimized using variable current source While $\mathrm{V}_{\mathrm{DC}}$ follows the $\mathrm{V}_{\mathrm{BAT}}$ with a fixed voltage. The proposed charger can also work in constant-voltage and constant-current modes for charging a Li-Ion battery [21, 22].

$$
\eta_{1} \cong \frac{V_{B A T} I_{C H}}{V_{D C}\left(I_{q}+I_{C H}\right)} \cong \frac{V_{B A T}}{V_{D C}+0.3}
$$

The dissipated power plays an important role in the performance of the battery charger. This power can be calculated by subtracting the power output and the input power is shown in (3):

$$
P_{\text {diss }}=\left(P_{D C}-P_{B A T}\right)+P_{q}=\left(V_{D C}-V_{B A T}\right) * I_{C H}+P_{q}
$$

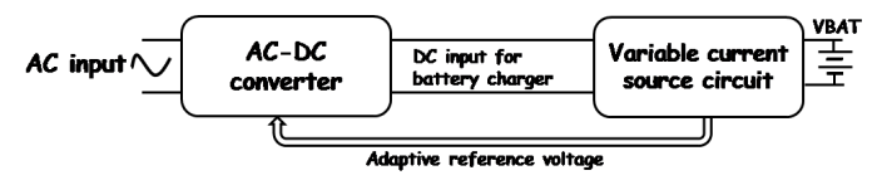

Figure 2. General architecture of the battery charger with a variable power source and a power control

\section{ARCHITECTURE OF THE PROPOSED BATTERY CHARGER}

\subsection{The Proposed Charge Mode Selection}

The charge mode selection is designed by a logic circuit, it is made by two analog comparators, logical ports such as NAND, OR, inverters and temperature detector circuit PTAT. First, the battery voltage is compared through comparators with the predefined references voltage $\left(\mathrm{V}_{\mathrm{L}}, \mathrm{V}_{\mathrm{H}}\right)$, in order to generate three control signals $\left(\mathrm{V}_{\mathrm{TC}}, \mathrm{V}_{\mathrm{CC}}\right.$ and $\left.\mathrm{V}_{\mathrm{CV}}\right)$; their main role is to provide currents $\left(\mathrm{I}_{\mathrm{TC}}, \mathrm{I}_{\mathrm{LC}}\right.$ and $\mathrm{I}_{\mathrm{CV}}$ ) via the reference current generator as shown in Figure $3 . \mathrm{V}_{\mathrm{C}}$ represents the control signal that provides a charging current to each state of charge. The output of the port OR is used to stop the charge if the charging current is reduced to $0.05 \mathrm{C}$ or if PTAT circuit detects 115 degrees. The charge mode-selection function is presented in Table 1.

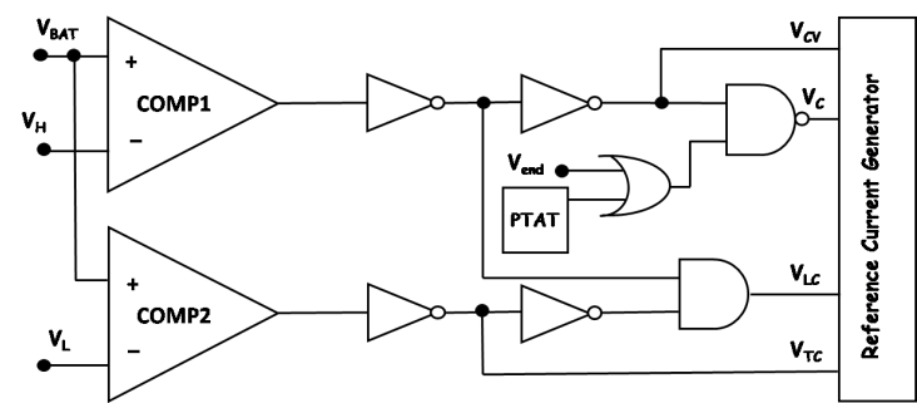

Figure 3. Schematic of the proposed charge mode selection 
Table 1. Charging mode control function

\begin{tabular}{ccccccc}
\hline Condition & VTC & VCC & VCV & VC & VEND & PTAT \\
\hline Constant Trickle Current & Hi & Lo & Lo & Hi & Lo & Lo \\
Constant large Current & Lo & Hi & Lo & Hi & Lo & Lo \\
Constant Voltage & Lo & Lo & Hi & Hi & Lo & Lo \\
End of charging & Lo & Lo & Hi & Lo & Hi & Hi \\
\hline
\end{tabular}

\subsection{Temperature detector PTAT}

Generally, all the techniques used in literature focus on rapidity, efficiency and life cycle of the Li-Ion batteries during the charging process. However, the major missed safety factor is the risk that can occur because of temperature, i.e. transistors temperature reaches a level that can damage the entire circuit. Therefore, we proposed a temperature detector PTAT circuit show in Figure 4 to control the charger temperature $[23,24]$.

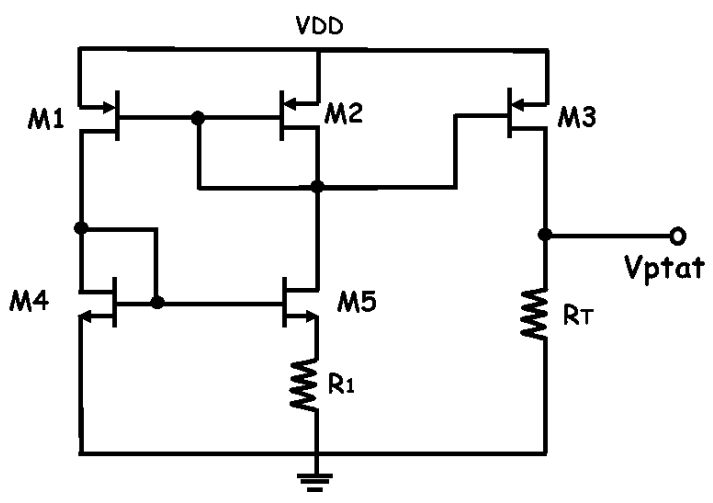

Figure 4. Schematic of the proposed temperature detector PTAT

The proposed PTAT based on current mirror structure is designed to stop the charging in any mode of charging once the temperature reaches $115^{\circ} \mathrm{C}$. The transistors M4 and M5 have a size ratio of 1: $\mathrm{N}$, and for M1 and M2 the same size. The current through the transistors M4 and M5 is given by (4):

$$
I_{D}=2 \eta \mu C_{o x} V_{T}^{2}(W / L) \exp \left[\left(V_{G S}-V_{T H}\right) / \eta V_{T}\right]
$$

where $\mathrm{V}_{\mathrm{T}}, \mathrm{V}_{\mathrm{GS}}$, and $\mathrm{V}_{\mathrm{TH}}$ represent the thermal voltage, gate-source voltage and threshold voltage respectively. $\mathrm{W} / \mathrm{L}$ is the width and length ratio, Cox represents the gate-oxide capacitance, $\eta$ is the substrate factor and $\mu$ is the carrier mobility. The PTAT voltage is the voltage over R1 which can be copied to the output and is given by (5):

$$
\Delta V_{G S}=\eta \frac{K T}{q} \ln N
$$

\subsection{Reference Current Generator}

The reference current generator is represented in Figure 5. This circuit is composed of a current block and a voltage block.

a. Current block

This block is used to produce the CC charging mode reference currents and the charge end current: The amplifier OP, the resistor $\mathrm{R}_{\mathrm{REF}}$ and the transistor M10 are used to generate a constant reference current $\mathrm{I}_{\mathrm{REF}}$, which is given by:

$$
I_{R E F}=\frac{V_{R E F}}{R_{R E F}}
$$


M1, M2, M3 and M4 represent the current mirror system in order to generate currents corresponding to each constant current ( $\mathrm{I}_{\mathrm{TC}}, \mathrm{I}_{\mathrm{LC}}$ and $\left.\mathrm{I}_{\mathrm{OFF}}\right)$, which are proportional to $\mathrm{I}_{\mathrm{REF}}$ as follow (7-9):

$$
\begin{aligned}
& I_{T C}=\frac{(W / L)_{2}}{(W / L)_{1}} I_{R E F} \\
& I_{L C}=\frac{(W / L)_{3}}{(W / L)_{1}} I_{R E F} \\
& I_{O F F}=\frac{(W / L)_{4}}{(W / L)_{1}} I_{R E F}
\end{aligned}
$$

b. Voltage block

The voltage block is used to produce the $\mathrm{CV}$ charge mode reference current. It is composed of current mirror (M5, M6) and a comparator, the charging current $\mathrm{I}_{\mathrm{CV}}$ is created as follows: As showing in Figure 5, when the battery voltage attains $4.2 \mathrm{~V}$ value, the current $\mathrm{I}_{\mathrm{CV}}$ is generated by the comparator in order to switch from $\mathrm{I}_{\mathrm{LC}}$ to $\mathrm{I}_{\mathrm{OFF}}$ (high level to low level). As already mentioned, each mode of charge has a corresponding current, which is generated by three transistors M9, M8 and M7 (controlled by $\mathrm{V}_{\mathrm{TC}}, \mathrm{V}_{\mathrm{LC}}$, $\left.\mathrm{V}_{\mathrm{CV}}\right)$.

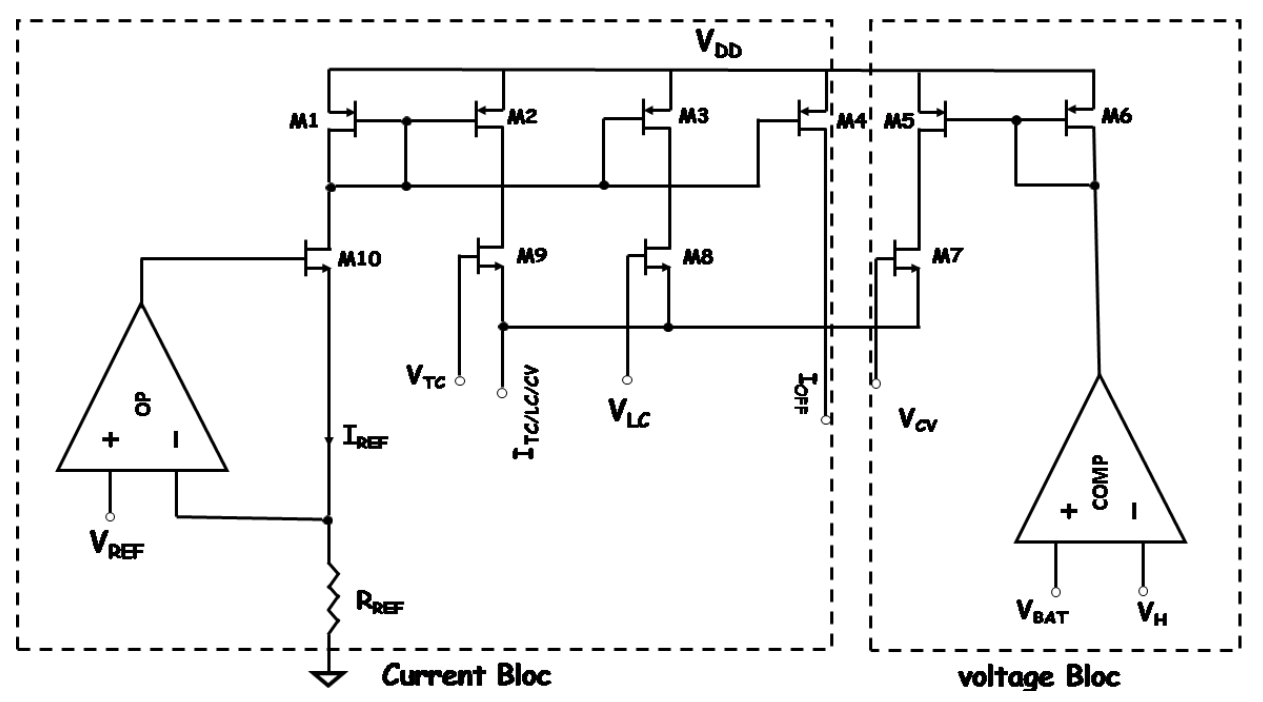

Figure 5. Schematic of reference current generator

\subsection{Charging current controller}

The purpose of the operating of charge current controller show in Figure 6 is to provide a driving voltage $\mathrm{V}_{\mathrm{D}}$ of the current source to each charging mode. The output current IS is measured by the current sensor in order to be compared with the three charging currents $\left(\mathrm{I}_{\mathrm{TC}}, \mathrm{I}_{\mathrm{LC}}\right.$ and $\mathrm{I}_{\mathrm{CV}}$ ) through the comparator M12-M15, the output voltage $V_{D}$ is changed with these input currents. In addition, it represents the lowest voltage level of the signal selector circuit (composed of the transistors M18 and M19).

As mentioned in section II, the stop procedure is activated by two method:

a. By the comparison of output current $\mathrm{I}_{\mathrm{S}}$ and reference current $\mathrm{I}_{\mathrm{OFF}}$ :

- If $I_{S}>I_{O F F}$ so $V_{E N D}$ is at the low level, which implies that the current voltage $V_{C}$ is at the high level.

- If $I_{S}<I_{O F F}$ than $V_{E N D}$ is at the higher level, which implies that the current voltage $V_{C}$ is at the low level. Knowing that the driving voltage $V_{D}$ is ordered by $V_{C}$, the current source is deactivated and the charging stops. 
b. By the temperature detected PTAT circuit:

The temperature of the charger is monitored throughout the charging period by a temperature detector. A reference voltage $\mathrm{V}_{\mathrm{TEP}}$ equivalent to 115 degrees is compared with the voltage of the state. The charging can be stopped in any state of charging according to the temperature of the circuit.

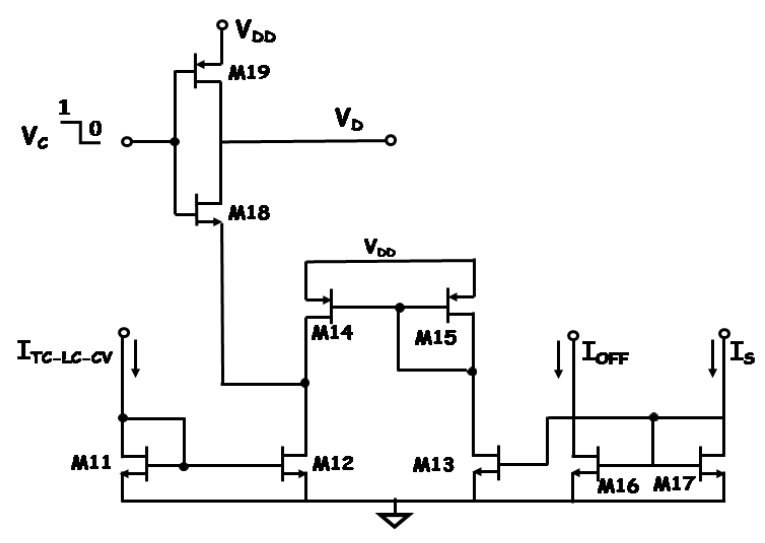

Figure 6. Schematic of charge current controller

\subsection{The proposed battery charger}

The proposed charging circuit with all blocks: Charging mode controller, reference current generator, charging current controller, temperature detector (PTAT) and finally current sensor circuit is represented in Figure 7. For the current sensor, the $\mathrm{M}_{\mathrm{S}}$ transistor plays the role of charging current sensor, and the current source represented by the power transistor $M_{P}$ which is used as a current source variable in order to generate a charge current to each charging mode.

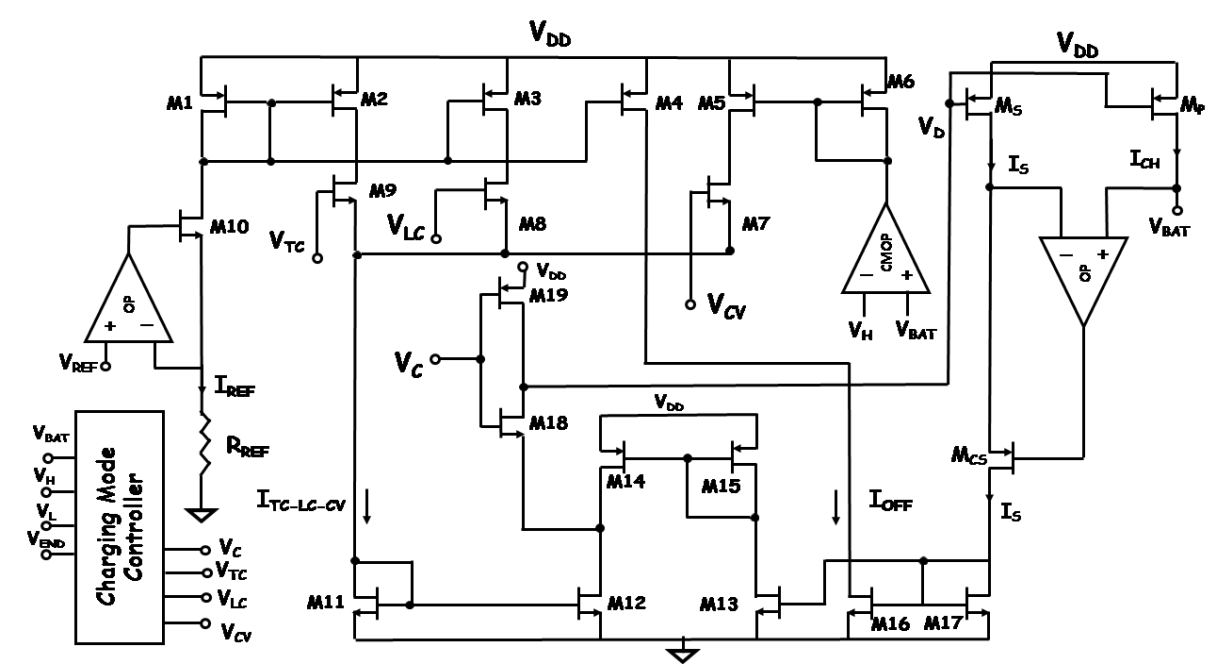

Figure 7. Circuit diagram of the proposed battery charger

\section{RESULTS AND DISCUSSIONS}

The proposed battery charger LDO-based is implemented in $180 \mathrm{~nm}$ CMOS TSMC technology. In order to study characteristics of our proposed charger, a testbench is showing in Figure 7 with an equivalent model of Li-ion battery. Based on the Li-ion battery model, the transient simulation of LDO-based battery charger is simulated with 2.7-4.5 V input voltage. The measurement results of our battery charger are shown in Figure 8, where the maximum current is respectively $280.5 \mathrm{~mA}$ and $1 \mathrm{~A}$ for trickle current mode and constant current mode. On the other hand, the maximum voltage is respectively $2.9 \mathrm{~V}$ and $4.22 \mathrm{~V}$ for trickle current mode and constant voltage mode. 


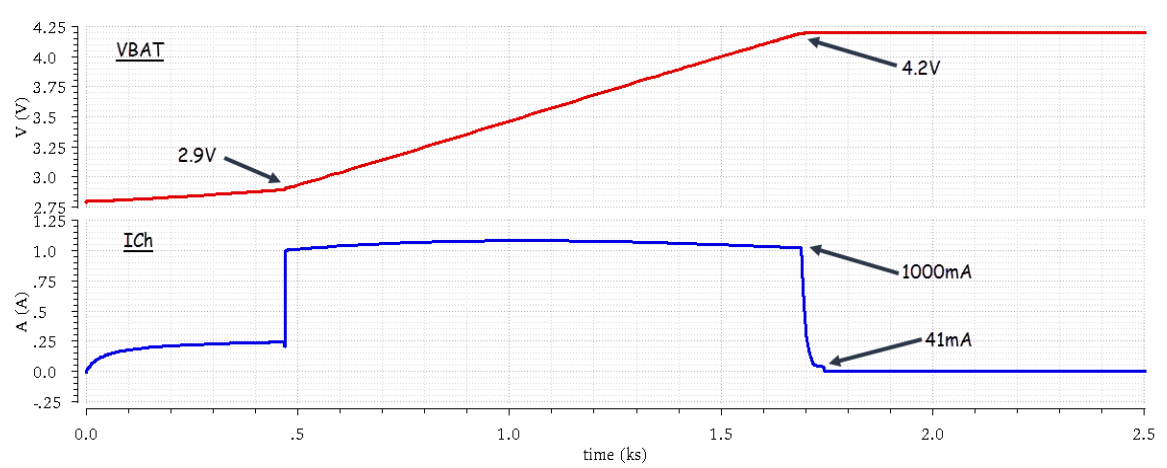

Figure 8. Simulation results of outputs the battery voltage Vbat and the charging current Ichg, respectively

Figure 9 presents the output voltages of the proposed charge mode selection. However, three signals $\mathrm{V}_{\mathrm{TC}}, \mathrm{V}_{\mathrm{LC}}$ and $\mathrm{V}_{\mathrm{CV}}$ are provided to control respectively trickle current, large current and constant voltage mode. As already mentioned in section 3, to have a complete protection system, it is necessary to integrate a temperature detector in order to protect the charger from any temperature damage. Figure 10 presents the simulation results of the proposed temperature detector PTAT. The charging process is done normally, once PTAT detects $115^{\circ} \mathrm{C}$ value the charging stops.

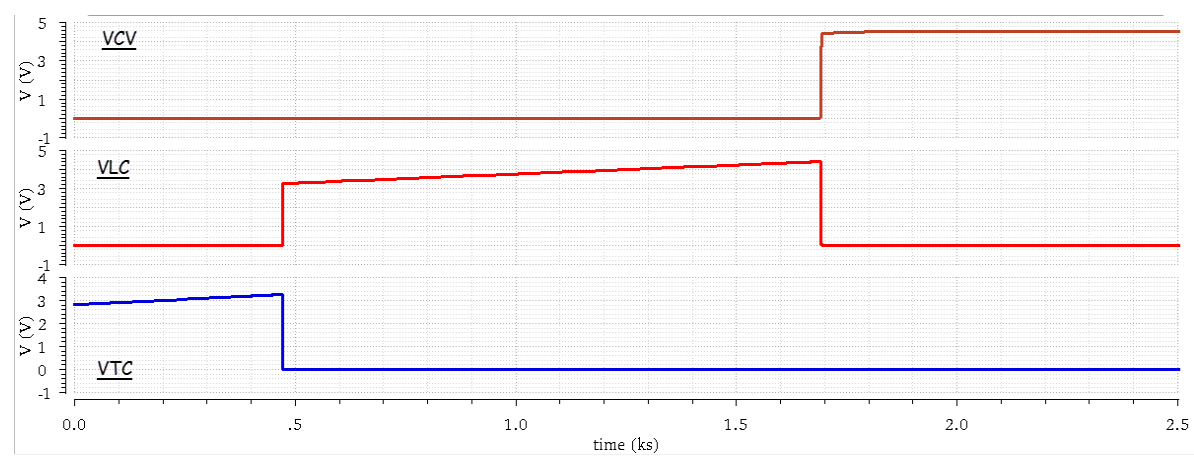

Figure 9. The outputs voltage of the proposed charge mode selection

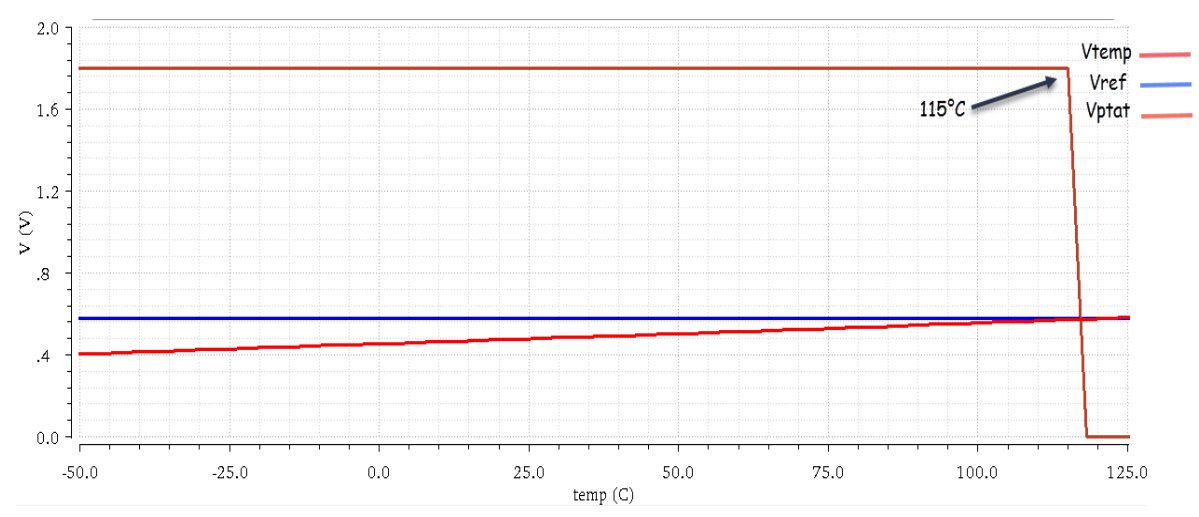

Figure 10. The simulation results of the proposed temperature detector PTAT

The power conversion efficiency of the proposed battery charger LDO-based is presented in Figure 11. The maximum efficiency of our charger is higher than the stated architectures [2, 3, 25]. As expected, the obtained results are much better compared to other works. However, the proposed techniques of charge modes-selection and temperature detector present excellent solutions: 
- To control the transition between the three modes of charge in order to reduce power dissipation and achieve high power efficiency (1A).

- To protect the battery from temperature damage.

Table 2 represents a performance comparison between our proposed work and others architecture of battery charger.

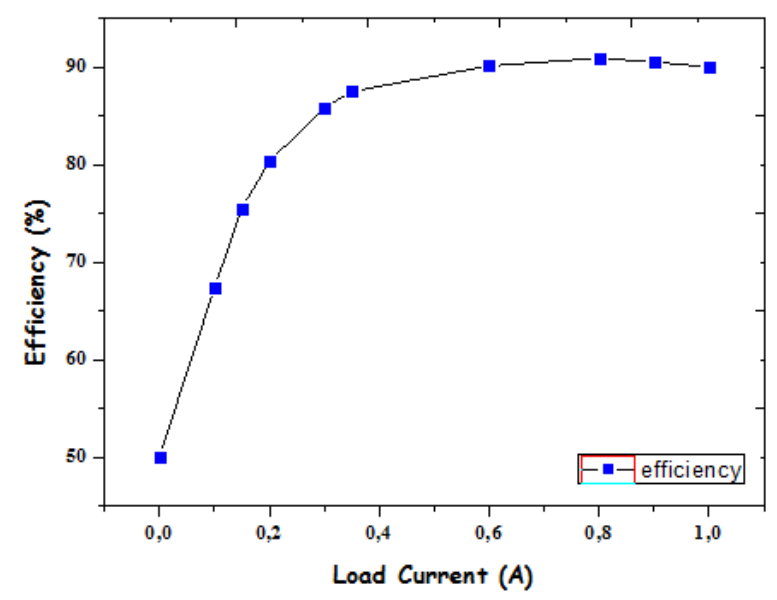

Figure 11. Power efficiency of the proposed battery charger

Table 2. Comparison of performances with other work

\begin{tabular}{ccccc}
\hline Parameters & {$[2]$} & {$[25]$} & {$[3]$} & This work \\
\hline Technology (um) & 0.18 CMOS & 0.35 CMOS & 0.35 CMOS & 0.18 CMOS \\
Power Supply (V) & $4.8-5$ & 4.4 & 4.5 & $2.7-4.5$ \\
Output voltage (V) & 4.2 & 4.1 & 4.2 & 4.2 \\
Maximum current (mA) & 448 & 1000 & 700 & 1000 \\
Efficiency (\%) & 87 & 68.3 & 70.9 & 90.9 \\
\hline
\end{tabular}

\section{CONCLUSION}

To conclude, the proposed architecture presents a good choice for phone applications as power management systems. Therefore, the proposed charger is able to switch from a charging mode to another without reducing its efficiency using the proposed charge selection, and also it can reach a high value of maximum charging current $(1000 \mathrm{~mA})$ in a short time, and with high power efficiency which can reach $90.9 \%$. The result of simulation of the proposed battery charger LDO-based confirms the high performance and high power efficiency of our architecture. The proposed charger is designed in $0.18 \mu \mathrm{m}$ CMOS TSMC technology, with a small area equal to $1.5 \mathrm{~mm} 2$.

\section{REFERENCES}

[1] D. Linden and T. B. Reddy, Handbook of Batteries, New York, NY, USA: McGraw-Hill, 2002.

[2] Y. Ziadi and H. Qjidaa, "A High Efficiency Li-Ion Battery LDO-Based Charger for Portable Application," Hindawi Publishing Active and Passive Electronic, pp. 1-9, 2015, Art no. 591986.

[3] H. M. Nguyen, L. D. Pham, and T. Hoang, "A novel Li-ion battery charger using multi-mode LDO configuration based on 350 nm HV-CMOS," Analog Integr Circ Sig Process, vol. 88, no. 3, pp 505-516, 2016.

[4] F. Farah et al., "New Analog Li-Ion Battery Charger Using Pulsed Charging Method," in Proceedings of 6th International Conference on Multimedia Computing and Systems (ICMCS), 2018, pp. 1-4.

[5] C. C. Tsai et al., "A multi-mode LDO-based Li-ion battery charger in $0.35 \mu \mathrm{M}$ CMOS technology," in Proceedings of the IEEE Asia-Pacific Conference on Circuits and Systems (APCCAS '04), Dec. 2004, vol. 1, pp. 49-52.

[6] H. Y. Yang et al., "An omnipotent Li-Ion battery charger with multimode controlled techniques," in Proceedings of the IEEE 10th International Conference on Power Electronics and Drive Systems (PEDS '13), 2013, pp. 531-534.

[7] K. El Khadiri and H. Qjidaa. "Li-Ion Battery Charging with a Buck-Boost DC-DC Converter for a Portable Device Power Management," Journal of Low Power Electronics, vol. 13, pp. 1-8, 2017.

[8] T. Thi et al., "A Wide Input Range Buck-Boost DC-DC Converter Using Hysteresis Triple-Mode Control Technique with Peak Efficiency of $94.8 \%$ for RF Energy Harvesting Applications," Energies, vol. 11, no. 7, p. 1618, 2018. 
[9] H. Wu et al., "Full-Range Soft-Switching-Isolated Buck-Boost Converters with Integrated Interleaved Boost Converter and Phase-Shifted Control," IEEE Trans. Power Electron, vol. 31, pp. 987-999, 2016.

[10] Jong-Seok et al., "High-efficiency peak-current-control non-inverting buck-boost converter using mode selection for single Ni-MH cell battery operation," Analog Integr Circ Sig Process, vol. 89, no. 2, pp. 297-306, 2016.

[11] Y. Tsai et al., "Digital Noninverting Buck-Boost Converter with Enhanced Duty Cycle-Overlap Control," IEEE Transactions on Circuits and Systems II, vol. 64, no. 1, 2016.

[12] Y. S. Hwang et al., "New Li-Ion Battery Charger Based on Charge-Pump Techniques," IEEE Trans, International Conference on Communications, Circuits and Systems, 2006.

[13] Hwang et al., "New compact Li-ion battery charger using charge pump techniques for portable appications," IEEE Journal of Solid-State Circuits, vol. 54, no. 4, 2007.

[14] Hwang et al., "New Li-ion batery charge based on charge pump technique," in IEEE International Conference on Comunication, Circuit and System, pp. 2259-2262, 2006.

[15] Pagano et al., "A 0.18 -lm monolithic Li-ion battery charger for wireless devices based on partial current sensing and adaptive reference voltage," IEEE Journal of Solid State Circuits, vol. 47, no. 6, pp. 1355-1368, 2012.

[16] C. H. Lin et al., "A Li-ion battery charger with smooth control circuit and built-in resistance compensator for achieving stable and fast charging," IEEE Transactions onCircuits and Systems, vol. 57, no. 2, pp. 506-517, 2010.

[17] Y. S. Hwang et al., "New compact CMOS Li-ion battery charger using charge-pump technique for portable applications," IEEE Transactions on Circuits and Systems I: Regular Papers, vol. 54, no. 4, pp. 705-712, 2007.

[18] K. H. Chen, "Extend the Battery Life by Highly Efficient DC-DC Converters," Proceedings Of 2004 Taipei Internal Power Forum, Dee, 2004.

[19] M. Chen and Gabriel A., "An Accurate Electrical Battery Model Capable of Predicting Runtime and I V Performance," IEEE Georgia Tech Analog and Power IC Design Lab, vol. 21, no. 2, 2006.

[20] L. Benini et al., "Discrete- time battery models for system-level low-power design," IEEE Trans. VLSI Systems, vol. 9 , no. $5,2001$.

[21] Jiann J. C. et al., "An omnipotent Li-ion battery charger with multimode control and polarity reversible techniques," International Journal of Electronics, vol. 103, no. 7, pp. 1138-1152, 2016.

[22] J. J. Chen et al., "A High-Efficiency Multimode Li-Ion Battery Charger with Variable Current Source and Controlling Previous-Stage Supply Voltage," IEEE transactions on industrial electronics, vol. 56, no. 7, 2009.

[23] A. Gołda and A. Kos, "Analysis and design of ptat temperature sensor in Digital cmos vlsi circuits," Proceedings of the International Conference Mixed Design of Integrated Circuits and System, 2006.

[24] J. V. De la Cruz and A. L. Aita, "A 1-V PTAT Current Reference Circuit with $0.05 \% / V$ Current Sensitivity to VDD," IEEE Trans International Symposium on Circuits and Systems, 2016.

[25] J. A. De Lima, "A compact and power-efficienct CMOS battery charger for implantable devices," in 27th Symposium on Integrated Circuit and System Design (SBCCI), Aracju, 2014. 